\title{
3 Impact of Operations management and advanced manufacturing technologies in Small Medium Enterprises to sustain in a market
}

Dr. Sandeep Kumar, Associate Professor, Department of Commerce and Management, Sarala Birla University, Ranchi.email: drsandeepkr1@gmail.com

\begin{abstract}
This paper seeks to explore the increased requirements for competitiveness, innovation, quality, flexibility, and information processing capability which has led a number of small and medium-sized enterprises (SMEs) to implement advanced manufacturing technologies (AMT) for sustainable development. It was found that while increased CSF and AMT assimilation levels directly impact operational performance in terms of increased productivity, cost reductions, flexibility, quality, and integration, a mismatch between the two significantly reduces performance. From an information processing view of the firm, it was also found that increased uncertainty in the SMEs' environment leads to increased CSF levels but not to increased assimilation of advanced manufacturing technologies in market.
\end{abstract}

Key words: SME, Operation Management,Manufacturing Technoligies, Market

\section{Introduction}

Increased requirements for competitiveness, innovation, quality, flexibility, and information processing capability has led many small and medium-sized enterprises (SMEs) have made sizable investments in adopting advanced manufacturing technologies (AMT) such as robotics and computer-aided manufacturing. They have also invested in implementing advanced computer-integrated manufacturing applications such as MRP II and ERP to plan, command, and control manufacturing resources and operations and link them with other organizational systems. Taken together, these technologies and applications constitute AMT that are assimilated to a varying degree in the SME's operational and managerial environment. Given the specificities than distinguish them from large enterprises in terms of environmental uncertainty, dependency, centralization, specialization, strategy, systems, resources, and flexibility, the management of manufacturing technology poses specific problems for SMEs. Thus is raised a fundamental question underlying these transformations, have small manufacturers actually 


\section{(C) Dr. Sandeep Kumar}

translated their technological investments into increased operational performance, be it in terms of reducing production costs, increasing the quality of goods produced, and customer service, or increasing the productivity, flexibility, and integration of manufacturing resources?

The present study focuses on the performance outcomes of alignment between the assimilation of AMT and the CSFs associated with operations and production management in manufacturing SMEs. Assimilation refers to the breadth and depth of a firm's proficiency in the use of AMT. Critical or key success factors are defined as the limited areas of activities that should receive constant and careful attention from management as satisfactory results in these areas should ensure performance.

\section{Background and development of the research model}

The notion of strategic alignment originates from the body of conceptual and empirical work in the organizational literature whose fundamental proposition is that organizational performance is the consequence of fit between two or more factors such as strategy, structure, and technology. The fundamental view of fit propounded by strategic management researchers and organization theorists was that it is a "dynamic search that seeks to align the organization with its environment and to arrange resources internally in support of that alignment". As strategy is the force that mediates between the firm and its environment, it is in practical terms the basic alignment mechanism, and the organizational technology must be well suited to it if a significant competitive advantage is to be created. Firms whose strategy and technology are aligned should be less vulnerable to external changes and internal inefficiencies, and should thus perform better because the technology provides the systems and processes necessary to successfully implement the strategy.

The idea that there is no one best way to manage an organization has been the underlying assumption of a great number of research models, in several areas of study. Organization theorists have focused on the study of contingency models that share the "underlying premise that context and structure must somehow fit together if the organization is to perform well". In strategic management, the general axiom of contingency theory is that no "strategy is universally superior, irrespective of the environmental or organizational context".

In examining the performance outcomes of implementing AMT and applications such as flexible manufacturing systems and manufacturing resources planning, Dean and Snell (1996) could not find 


\section{(C) Dr. Sandeep Kumar}

evidence to support a "best practice" approach. They thus argued for a contingency approach to the relationship between manufacturing practices and performance. Proposing in that the performance outcomes of AMT are contingent on the firm's strategic manufacturing goals, these authors found AMT to fit better with quality-oriented manufacturing strategies and less competitive environments. Other contingency models, which hypothesize that there is no best way to organize, have also been proposed and tested in the operations and technological management domain, be it for studying the alignment of the production administrative structure with the firm's environment and strategy, the contingency relationship between organizational structure and logistics strategy, the fit between new product strategies and control approaches, quality management practices in congruence with process choice and environmental uncertainty, and strategic and structural contingencies of adopting innovative manufacturing practices .

Information processing in the context of organizational decision making and uncertainty was used early on as a framework for a better comprehension of the fit between strategy, structure, and technology. Strategic change creates the need for more information and greater information gathering, interpreting, and synthesis capabilities, which in turn leads to changes in structure and technology. This approach is thus based on the assumption that organizations will be more effective when there is a match between the information-processing requirements of their strategy and the information-processing capacities of their overall structure.

An information processing view of the manufacturing firm emphasizes that organizational needs arise from having to operate in an environment, i.e. uncertain in terms of competitors, markets, and technology. As this environment becomes more hostile or complex, SMEs must increase their competitiveness by seeking new markets and putting emphasis on technological leadership and new product innovations. These needs are translated into a manufacturing strategy based on cost and/or differentiation that in turn determines operations management's objectives or CSFs that require increasing integration or flexibility, product variety, quality and precision, and information processing capabilities. From a contingency theory perspective, this view would also emphasize that the firm's manufacturing requirements must be matched by its technological capacity, expressed in terms of AMT, if performance is to be achieved. 
(C) Dr. Sandeep Kumar

\section{Operations management's critical success factors}

Although CSFs can be viewed at the industry level, implying that some CSFs are common to all organizations within industries, they can alternatively be viewed at the individual organization level, implying that different organizations may focus on different factors. An intermediate approach was taken here, whereby a number of generic Critical Success Factors were identified for small manufacturing firms that operate mainly in "make-to-order" mode. An organization's individual CSF level is determined on the basis of the relative importance placed upon the various factors. As presented in the Appendix, a comprehensive list of $26 \mathrm{CSFs}$ was developed from a review of the literature on operations and production management in SMEs and broken down into five categories, namely production planning and control, supply management, inventory management, quality management, and equipement management. However, prior research has shown that small and medium-sized manufacturers place varying degrees of emphasis on production planning and control, customer responsiveness, product quality, operational flexibility, productivity, and human resource management (HRM), and that this emphasis is related to their performance as "world-class" enterprises. Firms which place more importance on activities that are critical to operational performance (e.g. HRM activities) are also seen to be earlier adopters of "best practices" (e.g. developing a shared vision with employees), and thus should perform better.

\section{Advanced manufacturing technologies assimilation}

Encompassing a broad range of hardware and software-based technologies, AMT can be characterized not only by their rate of adoption but more importantly by their level of assimilation in organizations, as adoption by itself does not guarantee performance gains. It is thus important to differentiate between the adoption of a technology and its assimilation. In this regard, an organization's level of AMT assimilation defines the extent to which various technologies and applications have been mastered, and to what extent these form a coherent, integrated .Researchers have been interested in precisely defining AMT, in identifying determinants of AMT assimilation, and in the success or performance impacts of these technologies. There have been a number of empirical studies that have examined the actual benefits or performance outcomes from the implementation of AMT. The impacts that have been empirically confirmed include greater flexibility of the productive apparatus, greater quality, and variety of products, reduction in costs (notably in manpower costs), increases in productivity, reduced time-to-market, shorter production cycles and better response times, better information, better production planning, control and decision making, and increased performance in terms of service levels, customer satisfaction, market 
share, and financial returns .For SMEs, a tentative link between the use of such technologies and organizational performance has been established by a few researchers. Specifically, small firms that used computer-based forecasting and aggregate planning were found to have better financial performance (Riggs and Bracker, 1986), whereas those that had adopted technologies and applications such as CAM, robotics, computer-based production planning, and MRP showed an increased production output, reduced lead times, and greater profitability). In the same vein, SMEs that had available resources needed to adopt new manufacturing technologies and had in fact implemented such technologies were found to be more prevalent among high-growth firms.

\section{Alignment between critical success factors and AMT assimilation}

Structural contingency theory suggests that performance should increase when there is a fit between a firm's use of AMT and dimensions of the organizational context in which this use takes place. Based on this theoretical perspective, Kotha and Swamidass (2000) found empirical support for the premise that the fit between strategy and AMT is associated to superior performance. Gupta et al. (1998) also found organizational commitment to play a moderating role in the relationship between AMT intensity and performance.

As mentioned previously, AMT are deemed to provide both strategic and operational benefits to SMEs. These firms must, however, make significant investments to acquire an advanced manufacturing capability. The potentially high performance outcomes of AMT in conjunction with the importance of the human and financial resources required to implement such technologies necessitate that the organization align its manufacturing capability with its requirements (Das and Narasimhan, 2001). In other words, increasing importance placed upon its CSFs drives the manufacturing SME to increase its level of AMT assimilation. Hence, there should be greater performance when the SME achieves a level of AMT assimilation that matches its CSFs.

The "matching" perspective of fit is adopted as it is the most relevant to the theoretical foundations of this study. Here, fit is a theoretically defined match between two variables "without reference to a third criterion variable, although, subsequently, its effect on a set of criterion variables could be examined" (Venkatraman, 1989, p. 430). Adopting this perspective in an operations management context, one would state that fit exists when the firm's level of AMT assimilation matches the level of its CSFs. Whether the match improves firm performance can then be tested. One analytical scheme for supporting the matching 


\section{(C) Dr. Sandeep Kumar}

perspective is deviation score analysis, using the absolute difference between the standardized scores of the two variables (Alexander and Randolph, 1985). The hypothesized performance effects of fit are thus specified by the following equation:

\section{Environmental uncertainty}

Fighting to survive and prosper in markets that are ever more dynamic, unstable, and competitive, firms must react to increasing uncertainty in their environment. In strategic management and organization theory, the concept of environmental uncertainty is critical in the explanation of the strategy and its impact on performance. This concept has also been used as an explanatory factor of a firm's manufacturing strategy and strategic objectives such as flexibility and integration.

In regards to the firm's environment, it is the managers' perceptions that are more important than the actual environment in the sense that the decisions made by these individuals are designed in relation to the level of uncertainty perceived by them. As CSFs represent the few areas in which the firm must perform exceedingly well, they are obviously environmentally contingent. Returning to an information processing view of the firm, greater concentration on these areas can be seen as a way to cope with uncertainty and manufacturing SMEs may thus give more attention to their CSFs when they perceive their environment to be more uncertain.

Previous conceptual and empirical studies have proposed and attempted to identify environmental, organizational and individual determinants of AMT adoption, and successful implementation for both large and small firms. This research indicates that firms vary considerably in their capacity to assimilate, integrate, and leverage the business value of advanced technology, a potential reason being the varying degree of uncertainty that these firms have to contend within their business environment.

For organization theorists, environmental uncertainty has long been assumed to play an important role in technology-structure relationships. Increased instability in the environment is also seen as causing information acquisition to be more continuous, variant, and wide ranging, thus requiring more information processing capacity . A turbulent environment may induce firms to a more extensive use of information systems, in particular to counter forces in their industry such as the bargaining power of suppliers and customers. In a similar manner, it has been posited that the more uncertain the environment, the more manufacturing technology should be flexible and managers alert to adapt 


\section{C) Dr. Sandeep Kumar}

manufacturing systems to external changes. One could thus surmise that small manufacturers would react to increased pressures in their business environment by assimilating AMT to a broader and deeper extent.

\section{Methodology}

A survey questionnaire was mailed to the production manager of 700 manufacturing SMEs, randomly chosen from a directory of manufacturing firms. The latter manager is most knowledgeable on the firm's manufacturing practices and well-informed on its use of AMT. A total of 118 completed questionnaires were returned, thus a 16 percent response rate, i.e. relatively typical for small business survey research.

Environmental uncertainty was measured by using an instrument validated in a small business, in which the respondent is asked to indicate on five-point Likert scales the degree of change and unpredictability in the firm's markets, competitors, and production technology. CSF levels are assessed by asking the production managers to rate the importance of each factor in regard to their firm's success. AMT assimilation is measured by assessing the organization's proficiency or mastery level for each of the installed technologies and applications. Operational performance is assessed by having the production manager evaluate the achievement level of nine organizational goals in terms of productivity (e.g. reduce time-to-market), quality (e.g. increase product quality) and costs (e.g. reduce production downtime). It presents the results of a principal component analysis (with varimax rotation) of the 19 items used to measure the five dimensions of operational performance, confirming the validity of each dimension.

In testing the relationships hypothesized by the research model, structural equation modeling was used. The partial-least-squares (PLS) method was chosen for its robustness, in that it does not require a large sample or normally distributed multivariate data.

\section{Results}

As expected, the most prevalent manufacturing application is inventory management. One must note, however, that computer-based production scheduling has been implemented in less than one-third of the firms. The more advanced applications that integrate parts or all of the SME's value chain (MRP, MRP II or ERP) and link it with its business partners (EDI) are also not very prevalent, being implemented in less than 30 percent of the sampled firms, as is the case for the more advanced technologies. This would indicate that the more "soft" aspects of AMT, linked to the effective implementation and use of manufacturing resources management applications are more problematical for SMEs. 


\section{(C) Dr. Sandeep Kumar}

\section{Implications}

The results of this study have implications for researchers as well as for managers of small and mediumsized manufacturing firms. Built on the work of Ketokivi and Schroder (2004b) and extending this line of research to the small business area, these results provide further support for these authors' contention that the manufacturing practice-performance relationship is better explained by a strategic contingency argument than by a "best practice" argument. Given the previous empirical evidence on AMT in SMEs, our results confirm that one must not only look at the adoption rate or diffusion of AMT and applications. One must also look at the organizational assimilation of these technologies to obtain richer insights as to if and how small manufacturers benefit from AMT. In this regard, the concept of AMT assimilation as operationalized in this study seems promising, as we have found small firms to vary significantly in the degree to which they have mastered the various technologies and applications implemented. For instance, it is one thing to invest in industrial robots; it is another to be proficient in their use, and to use them in conjunction with other technologies such as CAD/CAM. Researchers wanting to further understand the actual benefits obtained by small firms from their implementation of AMT could thus use the technology assimilation concept in future studies in place of technology adoption.

Given the antecedent and contingency factors of AMT assimilation identified in this study, the basic link between manufacturing strategy and technology stands confirmed. How the firm perceives its competitive, commercial, and technological environment conditions its manufacturing objectives. In this regard, identifying a mismatch between the firm's CSFs and its level of AMT assimilation should trigger corrective action. One must also consider the new realities of global competition in which large and small firms work together within networks. Managers of small firms who enter into long-term agreements with prime contractors or who act as subcontractors for a few large customers must be prepared to increase their firm's level of AMT assimilation under pressure from their business partners for higher quality, better coordination of supply, production and distribution processes, and better information for planning and decision making. To this effect, acquiring new manufacturing technologies also implies investments in the training and organizational development required to assimilate these technologies. While these investments may be quite large and induce major changes in production processes, they should be transformed into increased operational performance for the SMEs that make them. 


\section{(C) Dr. Sandeep Kumar}

To the extent that small business managers want to augment their firm's manufacturing flexibility, reduce costs, improve quality and increase integration, the empirical evidence provided by this study leads one to conclude that they should look at their firm's present level of AMT assimilation in conjunction with their strategic intent. Identifying which technologies and applications are well assimilated and which are not would be essential in determining to what extent the firm's technology is aligned with its competitive environment and manufacturing objectives. For instance, this would help in answering a question posed to many small manufacturers today, i.e. if they require fully-integrated computer manufacturing (CIM) or enterprise (ERP) systems.

\section{Limitations and concluding remarks}

This investigation also has limitations that must be mentioned. Common to survey studies, the nature of the sample and perceptual nature of certain measures impose care in generalizing the results of the study. Adding objective measures of operational performance would also reinforce research findings on the impacts of AMT. There are obviously many factors other than those included in the research model that can influence the operational performance of manufacturing SMEs. This includes the evolving nature of buyer-supplier relationships as many manufacturing SMEs now operate within networks of cooperating firms. Future research should thus examine the performance outcomes of the fit between manufacturing strategy and technology in a more systemic perspective, i.e. by investigating the operations management structure and infrastructure as components that must be dynamically aligned with the evolving environment and business strategy of the firm if it is to achieve its manufacturing mission.

AMT are bound to take added importance for many SMEs that are facing the new challenges in terms of survival, growth, and competitiveness. Given the dearth of empirical knowledge in this regard, the present study has contributed to a better understanding of the nature and state of AMT assimilation in small manufacturing firms. In conjunction with the CSFs of operations management in these organizations, added knowledge has also been gained on the way in which this assimilation impacts operational performance.

It is recognized that SMEs are highly flexible and adaptable to change, be it environmental, operational or technological. Some of these already possess highly developed manufacturing systems and, in a more complex business environment, must implement practices such as concurrent engineering, just-in-time, synchronous production, and agile manufacturing to improve their competitive position. Investments in 


\section{(C) Dr. Sandeep Kumar}

AMT cannot insure greater performance unless they are coherent with the competitive environment and strategic objectives of SMEs. To this end, these enterprises must increase their ability to manage both manufacturing and information technologies, and thus seek increased support from researchers and practitioners.

\section{References}

Agarwal, R., Tanniru, M. and Wilemon, D. (1997), "Assimilating information technology innovations: strategies and moderating influences", IEEE Transactions on Engineering Management, Vol. 44 No. 4, pp. 347-58.

Alexander, J.W. and Randolph, W.A. (1985), "The fit between technology and structure as a predictor of performance in nursing subunits", Academy of Management Journal, Vol. 28 No. 4, pp. 844-59.

Alpar, P. and Kim, M. (1990), "Microeconomics approaches to the measurement of information technology value", Journal of Management Information Systems, Vol. 7 No. 2, pp. 55-70.

Ariss, S.S., Raghunathan, T.S. and Kunnathar, A. (2000), "Factors affecting the adoption of advanced manufacturing technologies in small firms", Advanced Management Journal, Vol. 65 No. 2, pp. 14-21.

Armstrong, C.P. and Sambamurthy, V. (1999), "Information technology assimilation in firms: the influence of senior leadership and IT infrastructures", Information Systems Research, Vol. 10 No. 4, pp. 304-27.

Assael, H. and Keon, J. (1982), "Nonsampling vs. sampling errors in survey research", Journal of Marketing, Vol. 46 No. 2, pp. 114-23.

Bakos, J.Y. and Brynjolfsson, E. (1993), "Information technology, incentives, and the optimal number of suppliers", Journal of Management Information Systems, Vol. 10 No. 2, pp. 37-53.

Bart, C.K. (1999), "Controlling new products: a contingency approach", International Journal of Technology Management, Vol. 18, pp. 395-413.

Bartazzaghi, E. and Francesco, T. (1989), "The impact of just-in-time on production system performance: an analytical framework", International Journal of Production Management, Vol. 9 No. 8, pp. 40-62.

Citation: Dr. Sandeep Kumar (2020). Impact of Operations management and advanced manufacturing technologies in Small Medium Enterprises to sustain in a market, Asian Mirror-March 2020, 7(1):16-26. doi - 10.21276/am.2020.7.1.AN3 


\section{(C) Dr. Sandeep Kumar}

Barua, A. and Lee, B. (1997), "The information technology productivity paradox revisited: a theoretical and empirical investigation in the manufacturing sector", International Journal of Flexible Manufacturing Systems, Vol. 9 No. 2, pp. 145-66. 